\title{
Cesarean Section Due to Social Factors Affects Children's Psychology and Behavior: A Retrospective Cohort Study
}

\author{
Xiu-Yu Shi ${ }^{1+}$, Jing Wang ${ }^{1 \dagger}$, Wei-Na Zhang ${ }^{2}$, Meng Zhao ${ }^{1}$, Jun $\mathrm{Ju}^{3}$, Xiao-Yan $\mathrm{Li}^{4}$, Qian $\mathrm{Lu}^{1}$, \\ Bin Wang ${ }^{1}$ and Li-Ping Zou ${ }^{1,5 *}$
}

${ }^{1}$ Department of Pediatrics, The First Medical Center, Chinese PLA General Hospital, Beijing, China, ${ }^{2}$ Department of Pediatrics, Beijing Friendship Hospital, Capital Medical University, Beijing, China, ${ }^{3}$ Department of Pediatrics, Beijing Chao-Yang Hospital, Capital Medical University, Beijing, China, ${ }^{4}$ Department of Neurology, Capital Institute of Pediatrics, Beijing, China, ${ }^{5}$ Center for Brain Disorders Research, Capital Medical University, Beijing Institute for Brain Disorders, Beijing, China

\section{OPEN ACCESS}

Edited by:

Teodor T. Postolache,

University of Maryland, Baltimore,

United States

Reviewed by:

Zinoviy Gutkovich,

Post Graduate Center for Mental Health (PCMH), United States

Celine Corona,

Bronx-Lebanon Hospital Center,

United States

${ }^{*}$ Correspondence:

Li-Ping Zou

zouliping21@hotmail.com

†These authors have contributed equally to this work

Specialty section

This article was submitted to Children and Health

a section of the journal

Frontiers in Pediatrics

Received: 24 July 2020 Accepted: 30 December 2020 Published: 25 January 2021

Citation:

Shi X-Y, Wang J, Zhang W-N, Zhao $M$,

Ju J, Li X-Y, Lu Q, Wang B and

Zou L-P (2021) Cesarean Section Due to Social Factors Affects Children's

Psychology and Behavior: A Retrospective Cohort Study.

Front. Pediatr. 8:586957.

doi: 10.3389/fped.2020.586957
Background: Cesarean section (CS) use has reached a frequency well-above what is expected on the basis of obstetric indications. The large increase in CS use, often for nonmedical indications, is of concern given the risks for both women and children. Research about the influence of CS on children's behavior is not new, but most studies didn't differentiate CS due to social factors (such as fear of labor pain, auspicious dates, etc.) from CS with medical indications. Medical indications for CS include fetal distress and intrauterine hypoxia, which may also affect the mental and physical health of the children, thus be a confounding factor. In China, a significant proportion of women undergo CS because of social factors, which provides us a good model to study whether non-fetal triggered delivery will affect children's behavior. Thus, we assessed the impact of CS due to social factors on child psychology and behavior.

Methods: We conducted a retrospective cohort study. Children were divided into three groups according to delivery mode: vaginal delivery (VD), CS with medical indications, and CS due to social factors (also called as elective cesarean section, ECS). Parents or guardians were required to complete four rating scales of Chinese version [Conners' Parent Rating Scale (CPRS), Child Behavior Checklist-Parent Form (CBCLPF), Swanson, Nolan, and Pelham rating scale-Parent Form (SNAP-IV-PF), and Behavior Rating Inventory of Executive Function-Parent Form (BRIEF-PF)] on psychological and behavioral problems regarding their children.

Results: Among the 38,780 children aged 7-15 years, 29,103 (75.05\%) were delivered by VD and 9,677 (24.95\%) were delivered by CS (7,844 with medical indications; 1,833 by ECS). Ten covariates were found to significantly affect ECS. Four rating scales were used in this study: CPRS, CBCL-PF, SNAP-IV-PF, and BRIEF-PF. ECS affected child psychology and behavior in several aspects including inattention, hyperactivity/impulsivity, social problems, and executive dysfunction. Regarding to inattention, the ECS group had a higher SNAP-IV-PF inattention score $(P=0.03)$, compared with the VD group. Logistic multivariate stepwise regression analysis showed that in the ECS group, the ORs were 1.20 in the partially adjusted analyses of 
SNAP-IV-PF and CPRS. Regarding to social problems, ECS group had a higher CBCLPF score for the social problems category compared with the VD group $(P=0.0001)$. Kruskal-Wallis rank sum tests showed that the ECS group had higher BRIEF-PF scores regarding Working Memory $(P=0.04)$, and Organize $(P=0.01)$ compared with the VD group.

Conclusions: CS affected the offspring's psychology and behavior. After removing possible influence of medical indications, the effect of CS due to social factors on the offspring's psychology and behavior still exists.

Keywords: cesarean section, elective cesarean section, children, behavior, psychology

\section{INTRODUCTION}

Vaginal delivery (VD) is a natural process which is a culmination of full maturation of the fetus, however, delivery mode has been deeply influenced by social factors in the last few decades. Comparing to Western populations, Asian women are more likely to choose CS. This disparity could partly be attributed to racial characteristics and cultural beliefs. Data from the World Health Organization (WHO) reveal that $46 \%$ of births in China involved CS in 2007-2008 (1). High frequency of CS was also found in other countries. In 2018, Boerma et al. (2) reported that frequency of CS in the Latin America and Caribbean region was $44.3 \%$, in the Dominican Republic was $58 \%$. This is the highest rate worldwide and three times higher than the WHO's recommendation of $15 \%$. Historically, the primary reasons to perform CS included obstetric complications or serious maternal illness, in order to save the mother or fetus. More recently, a significant proportion of women undergo CS because of social factors, such as fear of labor pain, concerned about complications (urinary incontinence and lower quality of sex life after VD), misconception of CS being safer and faster than VD, and auspicious dates (3).

The high prevalence of CS-especially CS due to social factors has initiated a debate about whether CS is always appropriate and has drawn attention to the effects of CS on maternal health. Many studies $(4,5)$ have suggested that CS is associated with an increased risk of complications as well as substantial economic burden. CS also affects the offspring's health, with previous studies showing that CS is associated with asthma, allergic rhinitis, diabetes $(6,7)$ and childhood acute lymphoblastic leukemia (8), and it may also affect neurological development which may lead to neurodevelopmental disorders $(9,10)$. Neurodevelopmental disorders usually affect behavioral, learning, and cognitive abilities, which may reduce the quality of life of individuals and their families. For example, attention deficit hyperactivity disorder (ADHD) is the most common neurodevelopmental disorder in children and is characterized by attention difficulty, hyperactivity, and impulsivity. ADHD affects an estimated $5.9-7.1 \%$ of children and adolescents worldwide (11) and often persists into adulthood (12). ADHD both directly impairs health and is associated with anxiety, other mood disorders, antisocial behavior, and addiction (13). Furthermore, $\mathrm{ADHD}$ leads to lost productivity, high social costs regarding health care and education, and increased conviction rates (14). Talge et al. (15) found that children delivered by CS exhibited higher inattention and ADHD index scores compared with children delivered by VD, especially among males. A link between CS and increased odds of autism spectrum disorder (ASD) has also been suggested (16).

However, interpretation of previous results is limited by small sample and inclusion criteria. For example, most previous studies assessing the effects of CS included CS with medical indications and CS due to social factors. Medical indications for CS include fetal distress and intrauterine hypoxia, which may also affect the mental and physical health of the children. CS due to medical factors is sometimes difficult to prevent, however, prevalence of ECS can be potentially modified by public education. Thus, it's crucial to study the effect of ECS on child behavioral outcomes. In China, a significant proportion of women undergo CS because of social factors, which provides us a good model to study whether non-fetal triggered delivery will affect children's health. Focusing on this group appears to be more appropriate because this differentiates the effects of infant and maternal diseases from CS. Two recent studies reported conflicting results with regards to outcomes of the impact of ECS on childhood behavioral development. Kelmanson reported that children delivered by ECS are more likely to exhibit behavioral and emotional disturbances (17), whereas Li et al. found that children delivered by ECS are less likely to have emotional or behavioral problems (18). Considering the high prevalence of CS in China and conflicting outcomes, it is essential to further explore the long-term effects of CS, particularly CS due to social factors, on child psychology and behavior.

\section{METHODS}

\section{Study Participants}

With the help of the Hohhot City Board of Education and the Ethics Committee, students from the second grade of primary school to the third grade of junior high school (7-15 years old) (24 primary schools and 10 junior high schools) whose households were registered in Hohhot were recruited for this study. A total of 43,165 questionnaires were distributed, and 38,780 completed questionnaires (Figure 1). 


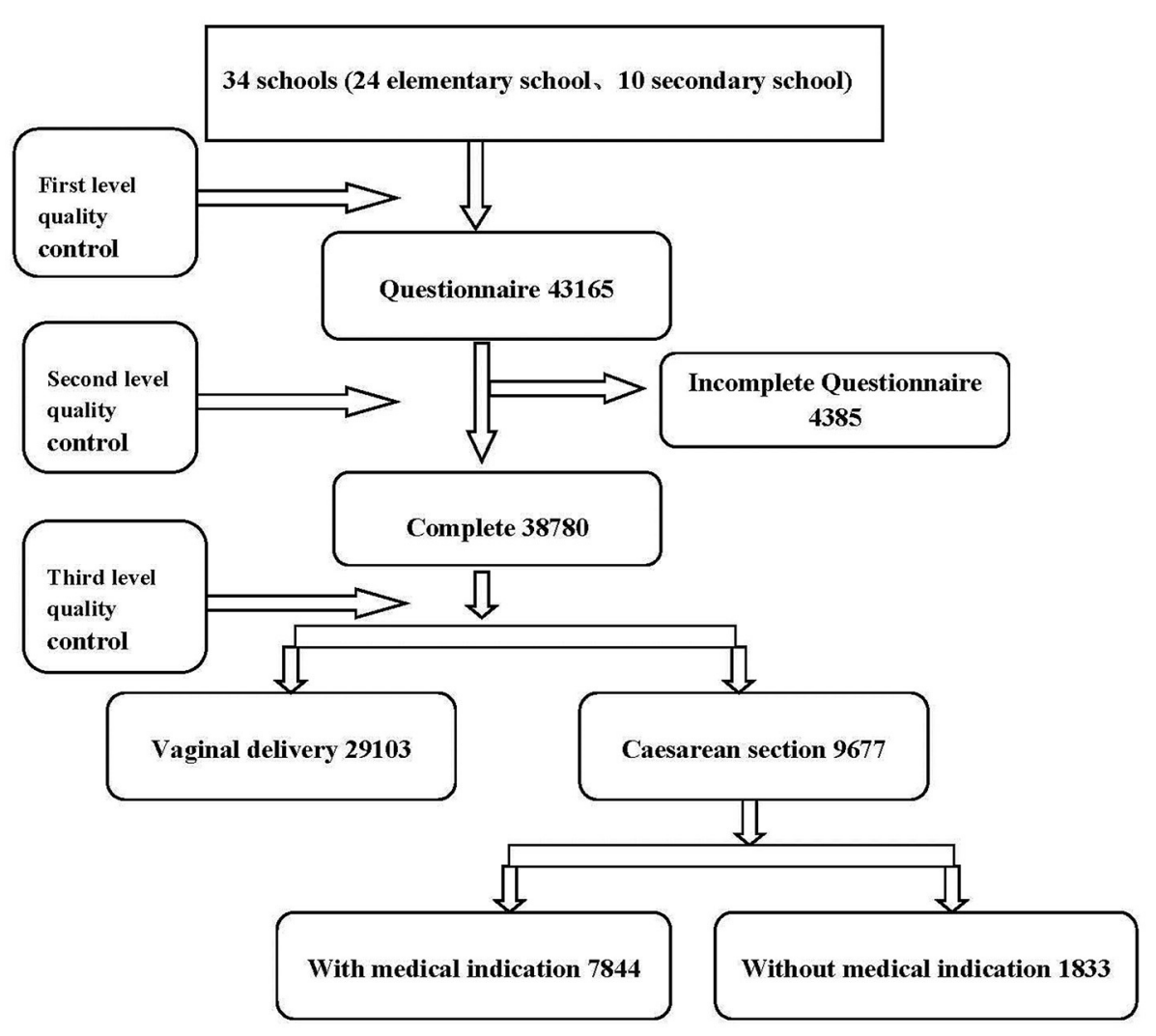

FIGURE 1 | Participant flow chart.

\section{Exposure Variable: Delivery Mode}

Delivery mode was divided into three categories: VD, CS with medical indications, and ECS. VD included spontaneous $\mathrm{VD}$, induced VD, and assisted VD with the use of forceps or vacuum extraction. Medical indications of CS included fetal distress, cephalopelvic disproportion, breech presentation, transverse lie position, maternal complications, previous CS, and other unspecified indications. ECS was defined as CS due to social factors (fear of labor pain, fear of hurt to baby, misconception of CS being safer and faster than VD, auspicious dates, and other).

\section{Covariates}

Covariates included parental occupations, parental ages, parental education levels, family type (two-parent or single-parent family), quality of family relationships, living conditions, habitable area, total household income; child's age, child's sex (male/female), physical examination, dietary habits, age at kindergarten enrollment; whether the mother had fever during pregnancy, whether abortion was considered, nutritional supplement use, complications, smoking, gestational age, birth weight, Apgar score, asphyxia, umbilical cord around the neck, and jaundice.

\section{Assessment of Child Psychopathology Conners' Parent Rating Scale (CPRS)}

CPRS (19) is a 48-item questionnaire based on a four-point (0, $1,2,3)$ scale according to symptom frequency. There are several subscale scores: conduct problems, impulsivity/hyperactivity, learning problems, anxiety, psychosomatic problems (including headache, stomach pain, other aches, vomiting or nausea, and bowel problems), and total scores.

\section{Child Behavior Checklist-Parent Form (CBCL-PF)}

CBCL-PF (20) contains 120 items on emotional, behavioral, and social problems reported by parents of children ages 4-16 years. Items related to eight syndrome categories (comprising anxious/depressed, withdrawn/depressed, somatic complaints, social problems, thought problems, attention problems, rulebreaking behavior, and aggressive behavior) are scored as 0 (not true), 1 (somewhat or sometimes true), or 2 (very true or often true) (21). Along with the total CBCL-PF score, scores were calculated for internalizing problems (withdrawn, somatic complaints, and anxious/depressed) and externalizing problems (rule-breaking behavior and aggressive behavior).

\section{Swanson, Nolan, and Pelham Questionnaire, 4th Version-Parent Form (SNAP-IV-PF)}

SNAP-IV-PF (22) includes 26 items based on a four-point $(0,1$, $2,3)$ scale according to symptom frequency. It consists of two 


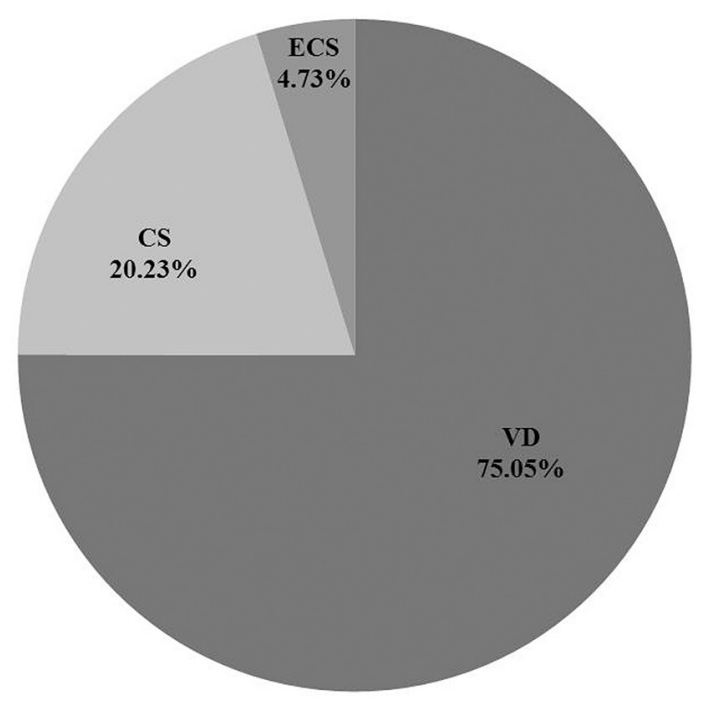

FIGURE 2 | Percentage of different delivery mode. CS, cesarean section with medical indications; ECS, cesarean section due to social factors; VD, vaginal delivery.

ADHD symptom subscales, one for inattention (nine items) and one for hyperactivity/impulsivity (nine items), and one subscale for oppositional defiant disorder (eight items). If the score on any of those subscales is 13 or above, it meets the threshold for ADHD diagnosis.

\section{Behavior Rating Inventory of Executive Function-Parent Form (BRIEF-PF)}

BRIEF-PF (23) is an 86-item questionnaire that is completed by the caregiver according to symptom frequency $(0,1$, 2). BRIEF-PF is designed to assess executive functioning in children and adolescents. It consists of the following subscales: Inhibit, Shift, Emotional Control, Working Memory, Plan/Organize, Organization of Materials, Monitor, and Initiate. These subscales yield three composite measures: Behavioral Regulation Index, Metacognition Index (MI), and Global Executive Composite. Higher BRIEF-PF scores indicate more severe executive function impairments.

\section{Statistical Analyses}

After examination of the completeness of the questionnaires, the questionnaire responses were coded, using EpiData software, we developed a CHECK program, and the data were independently inputted twice. To test the reliability and validity of the different rating scales, we used Split-half reliability and Pearson linear correlation analyses. Non-parametric (Kruskal-Wallis) rank sum tests were used for univariate analysis, and Logistic multivariate stepwise regression analysis was performed to control for confounding factors. All analyses were conducted using SAS 9.1 software (SAS Institute, Cary, NC, USA). Statistical significance was set at $P<0.05$.

\section{RESULTS}

\section{Distribution of Maternal and Child Characteristics Among the CS, ECS, and VD Groups}

A total of 43,165 questionnaires were distributed, 38,780 of which were valid (representing $89.84 \%$ of the questionnaires). Among the 38,780 children aged 7-15 years (birth years ranging from 1995 to 2003), 29,103 (75.05\%) were delivered by VD and 9,677 (24.95\%) were delivered by CS (7,844 with medical indications and 1,833 involving ECS, Figure 2). The distribution of maternal education, child's sex, child's age, living conditions, habitable area, and household income differed significantly by group $(P<0.05$, Table 1). Ten covariates were found to significantly affect ECS, including higher education, certain occupations (teacher, doctor, and public servant), single-parent family, maternal age, living in building, and etc. (Table 2).

\section{Rating Scale Reliability and Validity Reliability}

To assess response authenticity, participants were required to provide the delivery mode twice in the study questionnaire. The correlation coefficient for the correlation between the two answers was $0.993(P<0.0001$, Table 3). Four rating scales were used in this study: CPRS, CBCL-PF, SNAP-IV-PF, and BRIEF-PF. The split-half reliability of these scales was $0.76(0.70-0.80)$ and the Cronbach's alpha standard coefficient was 0.76 , suggesting that the reliability of each scale was acceptable and the overall consistency of each scale was good.

\section{Validity}

Pearson correlation analysis showed moderate to high correlations between SNAP-IV-PF and the other three rating scales (correlation coefficients $=0.50-0.73$, Table 4).

\section{Effect of ECS on Child Psychology and Behavior}

Non-parametric (Kruskal-Wallis) rank sum tests were used to evaluate the influence of ECS on child psychology and behavior. ECS affected four aspects: inattention, hyperactivity/impulsivity, social problems, and executive dysfunction. To verify the reliability of the results, stepwise multivariate logistic regression analysis was used to control for confounding factors. The results were similar to those of non-parametric (Kruskal-Wallis) rank sum testing, indicating that the results are accurate and reliable. Detailed results are listed below.

\section{Attention Deficit}

The SNAP-IV-PF scores regarding inattention and hyperactivity/impulsivity varied among children with different delivery modes. Based on Kruskal-Wallis rank sum testing, the ECS group had a higher SNAP-IV-PF inattention score $(P=$ 0.03 , Figure $3 \mathrm{~A}$ ), compared with the VD group, indicating that children delivered by ECS are more likely to exhibit inattention. Logistic multivariate stepwise regression analysis showed that in the ECS group, the odds ratio (OR) was 1.20 in the partially adjusted analysis (adjusting for child's age, child's sex, maternal 
TABLE 1 | Maternal and children characteristics according to mode of delivery.

\begin{tabular}{lccc}
\hline Characteristics & \multicolumn{3}{c}{ Mode of delivery } \\
\cline { 2 - 4 } & VD $n=$ & CS & ECS $n=$ \\
& $29,103(\%)$ & $n=7,844(\%)$ & $1,833(\%)$ \\
\hline
\end{tabular}

\section{Mother}

Education

University or higher

Junior college

Junior high school or

less

Single birth

Yes

No

\section{Child}

Gender

Male

Female

Age (years)

711

1215

Family

Family type

Traditional family

Single parent

Quality of family

relationships

Harmonious

Average or poor

Living conditions

Building

Bungalow

Habitable area (square

meters)

50

50

Household income

(Yuan/month)

3,000

3,000

$\begin{array}{lll}4,251(14.6) & 1,846(23.5) & 466(25.4) \\ & & \\ 12,863(44.2) & 4,136(52.7) & 989(54.0) \\ 11,989(41.2) & 1,862(23.7) & 378(20.6)\end{array}$

$<0.0001$

$(1,142.4)$

$11,989(41.2) \quad 1,862(23.7) \quad 378(20.6)$

$\begin{array}{lll}19,804(68.1) & 5,930(75.6) & 1,349(73.6) \\ 9,298(31.9) & 1,914(24.4) & 484(26.4)\end{array}$

$<0.0001$

(180.1)

$9,298(31.9) \quad 1,914(24.4) \quad 484(26.4)$

$14,019(48.2) \quad 4,143(52.8) \quad 928(50.6)$

$<0.0001$ (54.9)

$15,084(51.8) \quad 3,701(47.2) \quad 905(49.4)$

$16,703(57.4) \quad 5,093(64.9) \quad 1,302(71.0)$

$<0.0001$

(250.8)

$12,400(42.6) \quad 2,751(35.1) \quad 531(29.0)$

$$
\begin{array}{cccc}
28,438(97.7) & 7,674(97.8) & 1,773(96.7) & 0.02(8.3) \\
665(2.3) & 170(2.2) & 60(3.3) &
\end{array}
$$$$
\begin{array}{cccc}
26,535(91.2) & 7,233(92.2) & 1,680(91.7) & 0.01(8.6) \\
2,568(8.8) & 611(7.8) & 153(8.3) &
\end{array}
$$

$23,534(80.9) \quad 7,136(91.0) \quad 1,693(92.4)$

$<0.0001$

(567.9)

$5,569(19.1) \quad 708(9.0) \quad 140(7.6)$

$\begin{array}{llll}4,116(14.1) \quad 686(8.7) & 127(6.9) & <0.0001 \\ & & & (220.2)\end{array}$

$24,987(85.9) \quad 7,158(91.3) \quad 1,706(93.1)$

$12,394(42.6) \quad 2,744(35.0) \quad 553(30.2)$

$16,709(57.4) \quad 5,100(65.0) \quad 1,280(69.8)$

$V D$, vaginal delivery; CS, caesarean section with medical indications; ECS, cesarean section due to social factors.

education, family relationship, and only child) compared with the VD group $(P=0.003$, Figure 3B).

\section{Hyperactivity/Impulsivity}

SNAP-IV and CPRS were both used to assess impulsivity/hyperactivity. The Kruskal-Wallis rank sum test showed that the ECS group had a higher SNAP-IV-PF score compared with the VD group $(P=0.003$, Figure 3A). Logistic multivariate stepwise regression analysis showed that in the
TABLE 2 | Factors that affect ECS.

Factor $\begin{gathered}\text { Model } \\ \text { coefficient }\end{gathered} \chi^{2} \quad P \quad \begin{array}{r}\text { Odds ratio } \\ (95 \% \mathrm{Cl})\end{array}$

\section{Maternal factor}

University or higher

Junior college

Certain occupations ${ }^{1}$

coefficient

$(95 \% \mathrm{Cl})$

Age at delivery $\geq 35 Y$

\section{Family factor}

Live in building

Single-parent

0.893

$99.8<0.0001$

2.44

(2.05-2.91)

$0.689 \quad 106.5<0.0001 \quad 1.99$

(1.75-2.27)

$\begin{array}{llll}0.196 & 8.2 & 0.004 & 1.22\end{array}$

(1.06-1.39)

$\begin{array}{llll}0.255 & 7.3 & 0.007 & 1.29\end{array}$

(1.07-1.55)

$0.617 \quad 41.9<0.0001 \quad 1.85$

(1.54-2.23)

Single-parent

Household income ( $\geq 3,000$ Yuan/m)

0.247

1.28

(1.12-1.47)

Fetus factor

Cord around neck

Incipient abortion

$\begin{array}{ll}0.549 \quad 85.7<0.0001 & 1.73 \\ (1.54-1.95)\end{array}$

Birth weight $\geq 4 \mathrm{~kg}$

$\begin{array}{llll}0.311 & 11.7 & 0.0006 & 1.37\end{array}$

(1.14-1.63)

$\begin{array}{llll}0.286 & 6.4 & 0.01 & 1.33\end{array}$

$(1.07-1.66)$

section due to social factors.

Certain occupations ${ }^{1}$ refer to teacher, doctor, public servant.

TABLE 3 | Correlation test for the two answers to the mode of delivery.

\begin{tabular}{lcccc}
\hline Mode of delivery & $\begin{array}{c}\text { Frequency } \\
\text { (\%) } \boldsymbol{n}=\mathbf{3 8 , 7 8 0} \\
\text { (the } \\
\text { first answer) }\end{array}$ & $\begin{array}{c}\text { Frequency (\%) } \\
\boldsymbol{n}=\mathbf{3 8 , 7 8 0} \\
\text { (the second } \\
\text { answer) }\end{array}$ & $\begin{array}{c}\text { Correlation } \\
\text { coefficient }\end{array}$ & $\boldsymbol{P}$ \\
\hline VD & $29,103(75.0)$ & $29,134(75.1)$ & & \\
CS & $78,44(20.2)$ & $7,833(20.2)$ & 0.993 & $<0.0001$ \\
ECS & $1,833(4.8)$ & $1,813(4.7)$ & & \\
CS + ECS & $9,677(25.0)$ & $9,646(24.9)$ & & \\
\hline
\end{tabular}

$V D$, vaginal delivery; CS, cesarean section with medical indications; ECS, cesarean section due to social factors.

ECS group, the ORs were 1.20 in the partially adjusted analyses of SNAP-IV-PF and CPRS (adjusting for child's age, child's sex, maternal education, family relationship, and only child in the SNAP-IV-PF analysis; adjusting for child's age, maternal education, family relationship, only child, single-parent family, living conditions, and habitable area in the CPRS analysis) compared with the VD group $(P=0.003$, Figures 3B, 4B). These findings indicate that children delivered by ECS are more likely to be hyperactive/impulsive. 
TABLE 4 | Pearson correlation coefficient between different rating scales $(n=38,780)$.

\begin{tabular}{lcccc}
\hline & Conners & CBCL & SNAP-IV & BRIEF \\
\hline Conners & & 0.53 & 0.61 & 0.56 \\
CBCL & 0.53 & & 0.59 & 0.50 \\
SNAP-IV & 0.61 & 0.59 & & 0.73 \\
BRIEF & 0.56 & 0.50 & 0.73 &
\end{tabular}

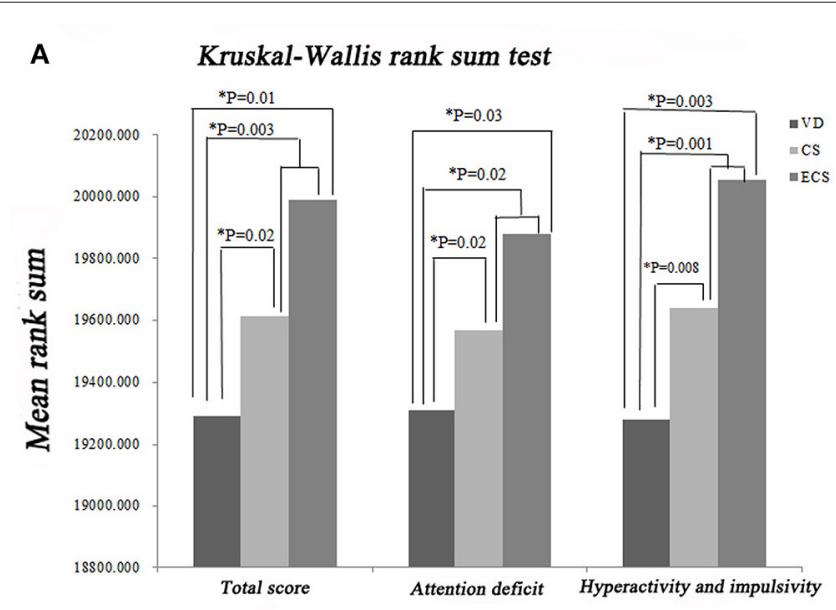

B Logistic multivariate stepwise regression analysis

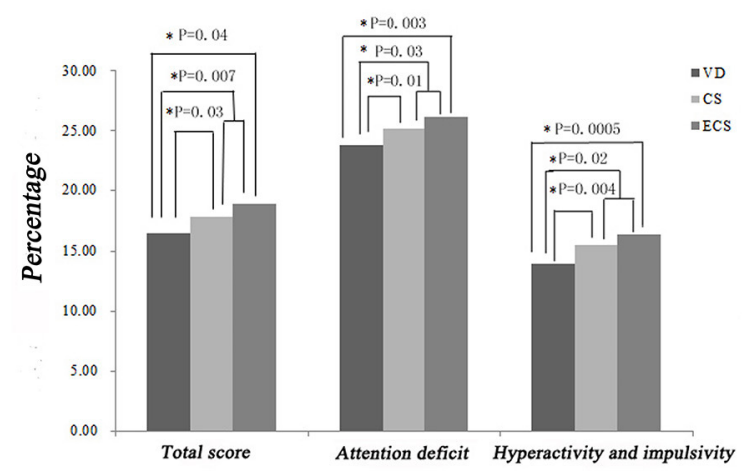

FIGURE 3 | Impact of ECS on child inattention and hyperactivity/impulsivity according to the SNAP-IV-PF rating scale. (A) Results of Kruskal-Wallis rank sum tests. (B) Results of stepwise multivariate logistic regression analysis. CS, cesarean section with medical indications; ECS, cesarean section due to social factors; SNAP-IV-PF, Swanson, Nolan, and Pelham questionnaire, fourth version; $\mathrm{VD}$, vaginal delivery.

\section{Social Problems}

Major manifestations of social problems include acting young, clingy behavior, difficulty getting along with others, experiencing teasing, and not being liked. Kruskal-Wallis rank sum tests showed that the ECS group had a higher CBCL-PF score for the social problems category compared with the VD group ( $P$ $=0.0001$, Figure 5A). Logistic multivariate stepwise regression analysis showed that in the ECS group, the OR was 1.30 in the partially adjusted analysis of the CBCL-PF social problems

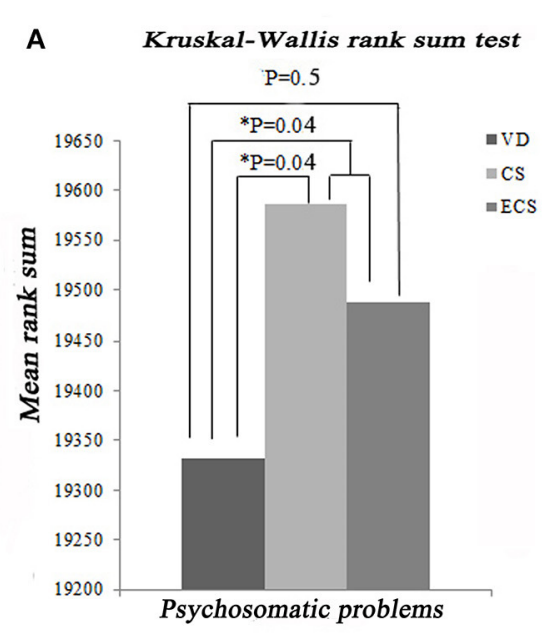

B Logistic multivariate stepwise regression analysis

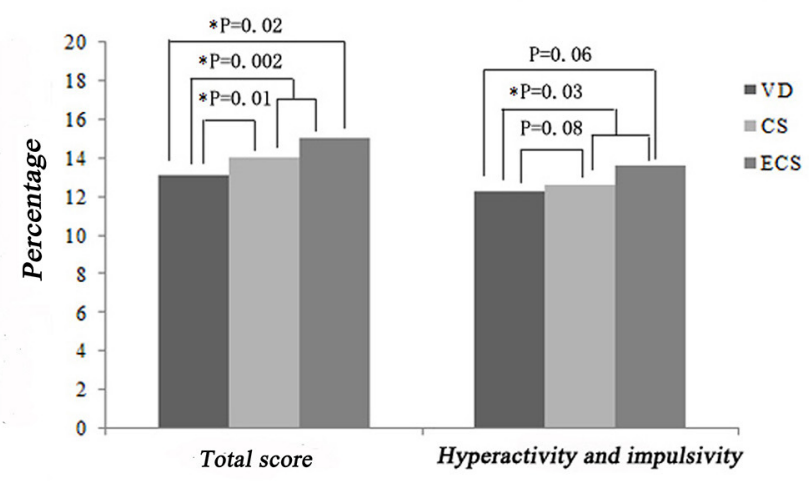

FIGURE 4 | Impact of ECS on hyperactivity/impulsivity and psychosomatic problems according to the CPRS. (A) Results of Kruskal-Wallis rank sum tests. (B) Results of stepwise multivariate logistic regression analysis. CPRS, Conners' Parent Rating Scale; CS, cesarean section with medical indications; ECS, cesarean section due to social factors; VD, vaginal delivery.

category (adjusting for child's age, child's sex, maternal education, family relationship, only child, single-parent family, living conditions, and habitable area) compared with the VD group $(P$ $=0.01$, Figure 5B).

\section{Executive Dysfunction}

Executive functions were assessed using multiple scores, including the BRIEF-PF BRI subscale score (Inhibit, Shift, Emotional Control, and Monitor) and the BRIEF-PF MI subscale score (Working Memory, Plan/Organize, Organization of Materials, and Task-Monitor). Kruskal-Wallis rank sum tests showed that the ECS group had higher BRIEF-PF scores regarding $\mathrm{MI}(P=0.04)$, Working Memory $(P=0.04)$, and Organize $(P=0.01)$ compared with the VD group (Figure 6A). Logistic multivariate stepwise regression analysis showed that in the ECS group, the ORs 1.20 in the partially adjusted analyses for MI $(P=0.04)$, Organize $(P=0.04)$, and Monitor $(P=0.01)$ (adjusting for child's age, maternal education, family relationship, only child, single-parent family, living conditions, and habitable 


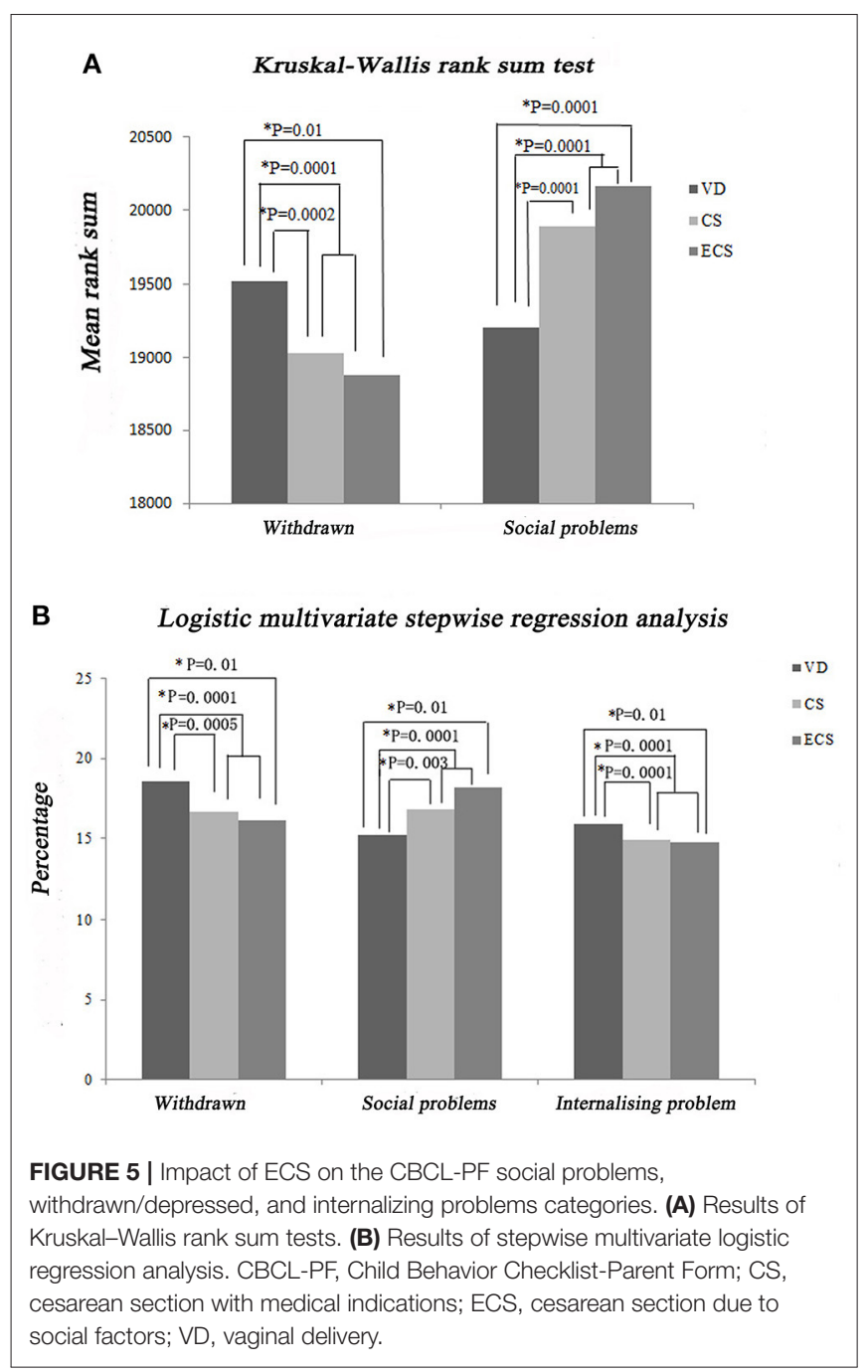

area) compared with the VD group (Figure 6B). Higher BRIEF$\mathrm{PF}$ scores indicate more severe executive function impairments.

\section{Psychosomatic Problems}

One of the CPRS subscales assesses psychosomatic problems including headache, stomach pain, other aches, vomiting or nausea, and bowel problems (constipation or diarrhea). The Kruskal-Wallis rank sum test showed that the CS + ECS group (involving CS with medical indications and ECS) had higher CPRS scores compared with the VD group $(P=0.04$, Figure 4A). However, there was no significant difference between the ECS and VD groups $(P=0.5)$, indicating that medical indications for CS resulted in psychosomatic problems rather than CS itself.

\section{DISCUSSION}

In the present study, we found that CS due to social factors accounted for $20 \%$ CS. Many factors including higher education, some occupations (teacher, doctor, and public servant), single-parent family, household income and living condition significantly affected the choice of CS. Among them, 33 women chose CS just because of auspicious dates. We further examined the associations between delivery mode especially CS due to social factors and child psychology and behavior in a large Chinese cohort. Stepwise multivariate logistic regression analysis indicated that CS due to social factors was associated with increased risk of psychological and behavioral problems (e.g., attention deficit, hyperactivity/impulsivity, social problems, and executive dysfunction) compared with VD, and this association persisted after controlling for multiple confounding factors. Even a small increase in the risk of psychological and behavioral problems may have large impacts on society.

Previous studies have reported short-term effects of CS on newborns including delayed lung maturation (24), poor thermogenesis (25), and reduced sucking reflex and breast feeding (26). There is increasing evidence indicating that CS also has long-term impacts on child physiology. Sevelsted et al. (27) reported associations between CS and asthma, systemic connective tissue disorders, juvenile arthritis, inflammatory bowel disease, and immune deficiencies. Moreover, the Childhood Leukemia International Consortium examined the association between CS and childhood leukemia in a large sample of cases and found that pre-labor CS was associated with acute lymphoblastic leukemia (8).

In addition to long-term impacts on child physiology, CS may be related to psychology. Curran et al. (16) conducted a systematic review of the literature to assess the impact of delivery mode on ASD and ADHD and found that, compared with VD, CS was associated with modestly increased odds of ASD and possibly ADHD. However, few studies have separately described the impacts of emergency CS and ECS on ASD or $\mathrm{ADHD}$. Emergency CS is performed when serious complications such as preeclampsia or fetal distress affect the mother and/or baby. These medical complications can have adverse impacts on child development. A recent study reported that the likelihood of child psychopathological problems was lowest in children born by ECS, followed by those born by spontaneous VD, whereas the highest probability was observed in those born by assisted VD (18). These findings were refuted by another study that reported that preschool children born by ECS may have increased risks of emotional disturbances and sleep problems (17). The contradictory results may be due to several study differences: some did not use population-based samples, some didn't differentiate ECS from CS with medical indications (28, 29) and did not adjust for confounders. The present study used a population-based sample and analyzed the data by non-parametric (Kruskal-Wallis) rank sum tests (for univariate analysis) and Logistic multivariate stepwise regression analysis (to control for confounding factors). Using these methods, CS due to social factors was associated with child psychological and behavioral problems.

There are several hypotheses to explain the potential relationship between CS and neurological development. First, newborns delivered by CS have different initial microbiota. Infants delivered by $\mathrm{VD}$ acquire bacterial communities resembling the maternal vaginal microbiota, and infants delivered by CS harbor bacterial communities similar to the 


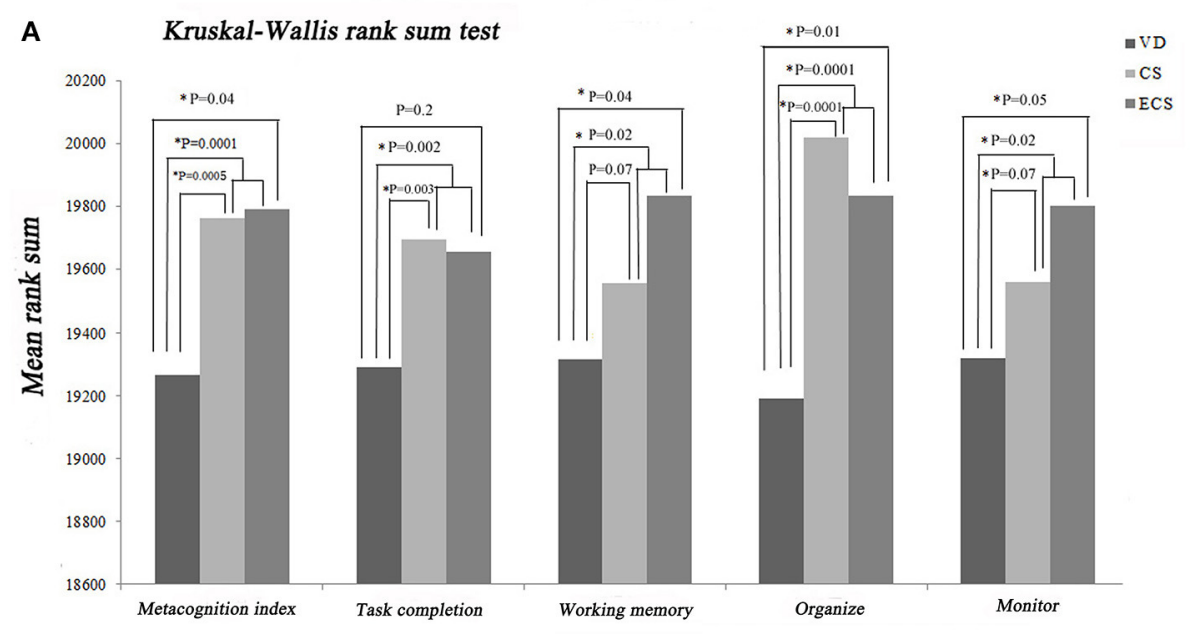

B Logistic multivariate stepwise regression analysis

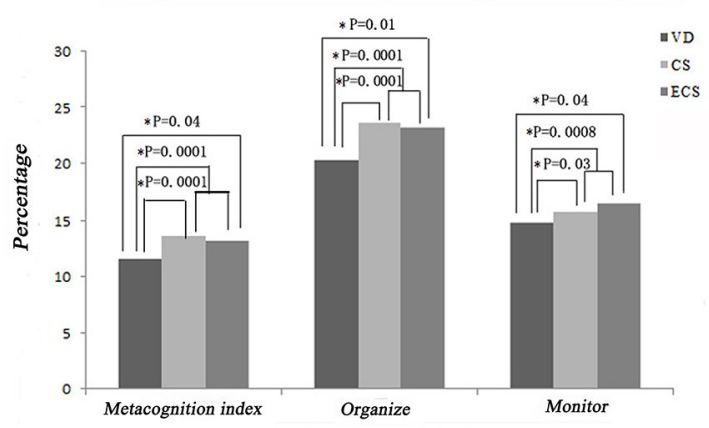

FIGURE 6 | Impact of ECS on childhood executive dysfunction according to the BRIEF-PF. (A) Results of Kruskal-Wallis rank sum tests. (B) Results of stepwise multivariate logistic regression analysis. BRIEF-PF, Behavior Rating Inventory of Executive Function-Parent Form; CS, cesarean section with medical indications; ECS, cesarean section due to social factors; VD, vaginal delivery.

skin microbiota (30). The composition of the core bacteria that we harbor during adulthood is mainly established in our first few years of life. There is a growing emphasis on the relationship between the gut microbiota and central nervous system disorders. The microbiota-gut-brain axis is composed of the brain, intestinal glands and other aspects of the gut, immune cells, and the gut microbiota. The immune system plays a key role in the microbiota-gut-brain axis (31). In an ASD mouse model created by prenatal valproic acid exposure, male offspring exhibited increased expression of neuroinflammatory markers, decreased serotonin, and disturbed social interactions compared with females (32). In another ASD mouse model created by maternal immune activation, offspring displayed both neurological and gastrointestinal symptoms associated with autism (33). Oral treatment with Bacteroides fragilis corrects gut permeability and resolved behavioral symptoms of ASD in offspring, suggesting that there are possible associations among the microbiota, immune system, and ASD (34). In addition, the stress of VD primes the hypothalamic-pituitary-adrenal axis and immune system, which enables offspring to deal with future insults (35). Furthermore, after ECS, the infant is separated from the mother after birth, which may have a negative impact on early bonding and attachment. Another possible explanation is that delivery by CS due to social factors is associated with changes in dopaminergic function and biochemistry (36), which alters how stress affects dopaminergic function.

There are several limitations to this study that need to be considered. Firstly, study didn't account for such potentially confounding variable as gestational age of the fetus, while gestational age in the case of ECS tends to be lower as obstetricians try to avoid spontaneous labor. Secondly, data analysis didn't differentiate the data related to the different types of VD such as assisted VD. However, despite this potentially obscuring factor associated with potential harm to the baby, statistical significance with regards to outcomes still remained. This study has several strengths. First, it is one of the largest observational studies to address the issue of effect of ECS on child psychology and behavior. Second, participants in the study were required to provide a delivery mode twice thus strengthening data on this key variable. Third, multiple rating 
scales and statistical methods are applied to measure the variables in this study.

Collectively, the evidence suggests that CS due to social factors is associated with increased odds of a wide spectrum of psychological and behavioral problems which may have large impacts on society. These findings improve our understanding of CS, help mothers make informed choices regarding the delivery mode and contribute to decreasing CS rate in China. We must keep in mind that everything has its own regular pattern, we should comply with it as much as possible. Further research is needed to confirm these findings in other cohorts and identify potential mechanisms.

\section{DATA AVAILABILITY STATEMENT}

The original contributions presented in the study are included in the article/Supplementary Material, further inquiries can be directed to the corresponding author/s.

\section{ETHICS STATEMENT}

Ethical review and approval was not required for the study on human participants in accordance with the local legislation and institutional requirements. Written informed consent to

\section{REFERENCES}

1. Lumbiganon P, Laopaiboon M, Gülmezoglu AM, Souza JP, Taneepanichskul S, Ruyan P, et al. Method of delivery and pregnancy outcomes in Asia: the WHO global survey on maternal and perinatal health 2007-08. Lancet. (2010) 375:490-9. doi: 10.1016/S0140-6736(09)61870-5

2. Boerma T, Ronsmans C, Melesse DY, Barros AJD, Barros FC, Juan L, et al. Global epidemiology of use of and disparities in caesarean sections. Lancet. (2018) 392:1341-8. doi: 10.1016/S0140-6736(1831928-7)

3. Zhang L, Zhang L, Li M, Xi J, Zhang X, Meng Z, et al. A cluster-randomized field trial to reduce cesarean section rates with a multifaceted intervention in Shanghai, China. BMC Med. (2020) 18:27. doi: 10.1186/s12916-020-1491-6

4. Yang XJ, Sun SS. Comparison of maternal and fetal complications in elective and emergency cesarean section: a systematic review and metaanalysis. Arch Gynecol Obstet. (2017) 296:503-12. doi: 10.1007/s00404-0174445-2

5. Sandall J, Tribe RM, Avery L, Mola G, Visser GH, Homer CS, et al. Shortterm and long-term effects of caesarean section on the health of women and children. Lancet. (2018) 392:1349-57. doi: 10.1016/s0140-6736(18)31930-5

6. Pistiner M, Gold DR, Abdulkerim H, Hoffman E, Celedón JC. Birth by cesarean section, allergic rhinitis, and allergic sensitization among children with a parental history of atopy. J Allergy Clin Immunol. (2008) 122:274-9. doi: 10.1016/j.jaci.2008.05.007

7. Thavagnanam S, Fleming J, Bromley A, Shields MD, Cardwell CR. A metaanalysis of the association between Caesarean section and childhood asthma. Clin Exp Allergy. (2008) 38:629-33. doi: 10.1111/j.1365-2222.2007.02780.x

8. Marcotte EL, Thomopoulos TP, Infante-Rivard C, Clavel J, Petridou ET, Schüz J, et al. Caesarean delivery and risk of childhood leukaemia: a pooled analysis from the Childhood Leukemia International Consortium (CLIC). Lancet Haematol. (2016) 3:e176-85. doi: 10.1016/S2352-3026(16)00002-8

9. Al Khalaf SY, O'Neill SM, O'Keeffe LM, Henriksen TB, Kenny LC, Cryan JF, et al. The impact of obstetric mode of delivery on childhood behavior. Soc Psychiatry Psychiatr Epidemiol. (2015) 50:1557-67. doi: 10.1007/s00127-015-1055-9

10. Curran EA, Khashan AS, Dalman C, Kenny LC, Cryan JF, Dinan TG, et al. Obstetric mode of delivery and attention-deficit/hyperactivity participate in this study was provided by the participants' legal guardian/next of kin.

\section{AUTHOR CONTRIBUTIONS}

L-PZ conceived the study. X-YS, JW, W-NZ, JJ, $\mathrm{X}-\mathrm{YL}, \mathrm{QL}$, and BW collected, interpreted the data and wrote the original draft of the manuscript. L-PZ reviewed and edited the manuscript. MZ performed the statistical analyses. All authors read and approved the final manuscript.

\section{FUNDING}

L-PZ was funded by grants from the National Key Research and Development Program of China (2016YFC1000707). X-YS was funded by the National Natural Science Foundation of China (81771389).

\section{ACKNOWLEDGMENTS}

The authors would like to thank Jun-Yin Wang (Allinpay Network Services Co., Ltd.), who helped us to transport the questionnaires. disorder: a sibling-matched study. Int J Epidemiol. (2016) 45:532-42. doi: 10.1093/ije/dyw001

11. Willcutt EG. The prevalence of $D S M-I V$ attention-deficit/hyperactivity disorder: a meta-analytic review. Neurotherapeutics. (2012) 9:490-9. doi: 10.1007/s13311-012-0135-8

12. Biederman J, Faraone SV. Attention-deficit hyperactivity disorder. Lancet. (2005) 366:237-48. doi: 10.1016/s0140-6736(05)66915-2

13. Biederman J. Attention-deficit/hyperactivity disorder: a selective overview. Biol Psychiatry. (2005) 57:1215-20. doi: 10.1016/j.biopsych.2004.10.020

14. Doshi JA, Hodgkins P, Kahle J, Sikirica V, Cangelosi MJ, Setyawan J, et al. Economic impact of childhood and adult attention-deficit/hyperactivity disorder in the United States. J Am Acad Child Adolesc Psychiatry. (2012) 51:990-1002.e2. doi: 10.1016/j.jaac.2012.07.008

15. Talge NM, Allswede DM, Holzman C. Gestational age at term, delivery circumstance, and their association with childhood attention deficit hyperactivity disorder symptoms. Paediatr Perinat Epidemiol. (2016) 30:17180. doi: $10.1111 /$ ppe. 12274

16. Curran EA, O'Neill SM, Cryan JF, Kenny LC, Dinan TG, Khashan AS, et al. Research review: birth by caesarean section and development of autism spectrum disorder and attention-deficit/hyperactivity disorder: a systematic review and meta-analysis. J Child Psychol Psychiatry. (2015) 56:500-8. doi: $10.1111 /$ jcpp. 12351

17. Kelmanson IAJEJoDP. Emotional and behavioural features of preschool children born by Caesarean deliveries at maternal request. Eur J Dev Psychol. (2013) 10:676-90. doi: 10.1080/17405629.2013.787024

18. Li HT, Ye R, Achenbach TM, Ren A, Pei L, Zheng X, et al. Caesarean delivery on maternal request and childhood psychopathology: a retrospective cohort study in China. BJOG. (2011) 118:42-8. doi: 10.1111/j.1471-0528.2010.02762.x

19. Goyette CH, Conners CK, Ulrich RF. Normative data on revised Conners Parent and Teacher Rating Scales. J Abnorm Child Psychol. (1978) 6:221-36. doi: 10.1007/BF00919127

20. Achenbach TM, Howell CT, Quay HC, Conners CK. National survey of problems and competencies among four- to sixteen-year-olds: parents' reports for normative and clinical samples. Monogr Soc Res Child Dev. (1991) 56:1131. 
21. Ivanova MY, Dobrean A, Dopfner M, Erol N, Fombonne E, Fonseca $\mathrm{AC}$, et al. Testing the 8-syndrome structure of the child behavior checklist in 30 societies. J Clin Child Adolesc Psychol. (2007) 36:405-17. doi: 10.1080/15374410701444363

22. Gau SS, Shang CY, Liu SK, Lin CH, Swanson JM, Liu YC, et al. Psychometric properties of the Chinese version of the Swanson, Nolan, and Pelham, version IV scale-parent form. Int J Methods Psychiatr Res. (2008) 17:35-44. doi: 10.1002/mpr.237

23. Gioia GA, Isquith PK, Guy SC, Kenworthy LJCN. Behavior rating inventory of executive function. Child Neuropsychol. (2000) 6:235-8. doi: 10.1076/chin.6.3.235.3152

24. Faxelius G, Hägnevik K, Lagercrantz H, Lundell B, Irestedt L. Catecholamine surge and lung function after delivery. Arch Dis Childh. (1983) 58:262-6. doi: $10.1136 /$ adc.58.4.262

25. Christensson K, Siles C, Cabrera T, Belaustequi A, de la Fuente P, Lagercrantz $\mathrm{H}$, et al. Lower body temperatures in infants delivered by caesarean section than in vaginally delivered infants. Acta Paediatr. (1993) 82:128-31. doi: 10.1111/j.1651-2227.1993.tb12622.x

26. Vestermark V, Høgdall CK, Birch M, Plenov G, Toftager-Larsen K. Influence of the mode of delivery on initiation of breast-feeding. Eur J Obstet Gynecol Reprod Biol. (1991) 38:33-8. doi: 10.1016/0028-2243(91) 90204-x

27. Sevelsted A, Stokholm J, Bønnelykke K, Bisgaard H. Cesarean section and chronic immune disorders. Pediatrics. (2015) 135:e92-8. doi: 10.1542/peds.2014-0596

28. Huh SY, Rifas-Shiman SL, Zera CA, Edwards JW, Oken E, Weiss ST, et al. Delivery by caesarean section and risk of obesity in preschool age children: a prospective cohort study. Arch Dis Childh. (2012) 97:610-6. doi: 10.1136/archdischild-2011-301141.

29. Goldani HA, Bettiol H, Barbieri MA, Silva AA, Agranonik M, Morais MB, et al. Cesarean delivery is associated with an increased risk of obesity in adulthood in a Brazilian birth cohort study. Am J Clin Nutr. (2011) 93:1344-7. doi: 10.3945/ajcn.110.010033

30. Dominguez-Bello MG, Costello EK, Contreras M, Magris M, Hidalgo G, Fierer $\mathrm{N}$, et al. Delivery mode shapes the acquisition and structure of the initial microbiota across multiple body habitats in newborns. Proc Natl Acad Sci USA. (2010) 107:11971-5. doi: 10.1073/pnas.1002601107

31. Luna RA, Foster JA. Gut brain axis: diet microbiota interactions and implications for modulation of anxiety and depression. Curr Opin Biotechnol. (2015) 32:35-41. doi: 10.1016/j.copbio.2014.10.007

32. de Theije CG, Wopereis H, Ramadan M, van Eijndthoven T, Lambert $\mathrm{J}$, Knol J, et al. Altered gut microbiota and activity in a murine model of autism spectrum disorders. Brain Behav Immun. (2014) 37:197-206. doi: 10.1016/j.bbi.2013.12.005

33. Barber EL, Lundsberg LS, Belanger K, Pettker CM, Funai EF, Illuzzi JL. Indications contributing to the increasing cesarean delivery rate. Obstet Gynecol. (2011) 118:29-38. doi: 10.1097/AOG.0b013e31821e5f65

34. Hsiao EY, McBride SW, Hsien S, Sharon G, Hyde ER, McCue T, et al. Microbiota modulate behavioral and physiological abnormalities associated with neurodevelopmental disorders. Cell. (2013) 155:1451-63. doi: 10.1016/j.cell.2013.11.024

35. Cho CE, Norman M. Cesarean section and development of the immune system in the offspring. Am J Obstet Gynecol. (2013) 208:249-54. doi: 10.1016/j.ajog.2012.08.009

36. Boksa P, El-Khodor BF. Birth insult interacts with stress at adulthood to alter dopaminergic function in animal models: possible implications for schizophrenia and other disorders. Neurosci Biobehav Rev. (2003) 27:91-101. doi: 10.1016/s0149-7634 (03)00012-5

Conflict of Interest: The authors declare that the research was conducted in the absence of any commercial or financial relationships that could be construed as a potential conflict of interest.

Copyright (c) 2021 Shi, Wang, Zhang, Zhao, Ju, Li, Lu, Wang and Zou. This is an open-access article distributed under the terms of the Creative Commons Attribution License (CC BY). The use, distribution or reproduction in other forums is permitted, provided the original author(s) and the copyright owner(s) are credited and that the original publication in this journal is cited, in accordance with accepted academic practice. No use, distribution or reproduction is permitted which does not comply with these terms. 